\title{
On the Zeros of Absorptive Diffraction Cross Sections
}

\author{
Jnanadeva Maharana \\ Institute of Physics, A/105, Saheed Nagar, Bhubaneswar-751007, India
}

\begin{abstract}
Distribution of the zeros of absorptive differential cross section is studied. Several rigorous bounds are deduced on the slope and curvature of diffraction peak. Energy dependence of the slope of diffraction peak and location of zeros of the cross section impose a constraint on ratio of curvature and slope of diffraction peak.
\end{abstract}

\section{Introduction}

The purpose of this note is to study the distribution of zeros of absorptive elastic differential cross sections and to deduce bounds on the slope and curvature of diffraction peak (whose precise mathematical definition will be given later). It is shown that the sufficient condition for scaling can be obtained if the zeros are distributed in a specific manner in the complex $\cos \theta$ plane, $\theta$ being the centre of mass scattering angle.

Let us briefly recapitulate some of the properties of two body scattering amplitude which follow from the assumptions of axiomatic quantum field theory $[1,2]$.

i) Analyticity in $s$ and $t$. The scattering amplitude $F(s, t)$ is analytic in the domain $\left(|t|<t_{0} \otimes\right.$ cut $s$-plane), where $t_{0}$ is some positive number (threshold for $t$-channel reactions). Here $s$, denotes the square of the centre of mass energy and $t$ the square of the momentum transfer.

ii) Polynomial Boundedness [1]. For $|t|<t_{0}$ and some constant $N, F(s, t)$ satisfies the inequality $|F(s, t)|<|s|^{N}$.

iii) Analyticity in the Lehmann-Martin Ellipse. For fixed real $s$ above threshold, $F(s, t)$ is analytic in an ellipse in the $\cos \theta$ plane with foci at $\cos \theta=+1$ and -1 and semimajor axis of the form $1+a / s$ for large $s$. Consequently, the partial wave expansion for $F(s, t)$ converges inside this ellipse. 
iv) The Froissart Bound [3]. The total cross section is bounded from above as

$$
\sigma_{t}(s) \leqq \frac{4 \pi}{t_{0}-\varepsilon}\left(\ln \left(s / s_{0}\right)\right)^{2}
$$

Here and everywhere $\varepsilon$ denotes an arbitrarily small positive number.

The distribution of zeros of the scattering amplitude has been studied for the general case by Auberson et al. [4] in connection with violation of the Pomeranchuk theorem. In [4], it was shown that when the Froissart bound is saturated that is $\sigma_{\text {tot }}(\ln s)^{-2} \rightarrow$ const, $s \rightarrow \infty$, then the function $f(s, \tau)$ $=F\left(s,-t_{0} \tau(\ln s)^{-2}\right) / F(s, o)$ has the limit

$$
f(s, t)=f(\tau)
$$

for at least one sequence $\left\{s_{n}\right\} \rightarrow \infty$. Furthermore, is an entire function of order $1 / 2$ and the forward slope $b(s) \simeq(\ln s)^{2}$. The scaling properties of the diffraction peak have been studied by several authors following the work of Auberson et al. [4].

Recently, it has been shown $[5,7]$ that the elastic differential cross sections for pure absorptive amplitudes are functions of positive type. This result holds good for external particles with arbitrary spin as a fundamental consequence of unitarity. Mahoux [7] has further shown that the elastic differential cross sections can be expanded in Legendre polynomials with positive coefficients. Thus

$$
\frac{d \sigma^{A}}{d t}(s, t) / \frac{d \sigma^{A}}{d t}(s, o)=\sum_{0}^{\infty}(2 n+1) \alpha_{n}(s) P_{n}(\cos \theta),
$$

where $\frac{d \sigma^{A}}{d t}(s, o)$ is the differential cross section in the forward direction and the coefficions $\alpha_{n}(s) \geqq 0$. Here the superscript $A$ denotes that the cross section is purely absorptive. Recently, Roy [8] has extended this result to inelastic reactions.

\section{Bounds on Slope of Diffraction Peak}

Let

$$
f(s, t) \equiv \frac{d \sigma^{A}}{d t}(s, t) / \frac{d \sigma^{A}}{d t}(s, o) .
$$

The function $f(s, t)$ has following properties;

a) $f(s, o)=1$.

b) $|f(s, t)| \leqq f(s, o)=1$ due to positivity of $\alpha_{n}(s)$.

c) For complex values of $|t|<t_{0}, f(s, t)$ is founded from above as

$$
\mid f(s, t)<\exp \left[L(s)\left(|t| /\left(t_{0}-\varepsilon\right)\right)^{1 / 2}\right]
$$

where $L(s)=\ln \left(s^{2} / \frac{d \sigma^{A}}{d t}(s, o)\right)$.

Note that is follows from the Froissart bound and the Jin, Martin lower bound that $2 \ln s \leqq \ln \left(s^{2} / \frac{d \sigma^{A}}{d t}(s, o)\right) \leqq 14 \ln s$. 
The bound (5) is due to Auberson and Roy [6]. We define

$$
\bar{s}=s^{2} / \frac{d \sigma^{A}}{d t}(s, o) \text {. }
$$

Now we proceed to derive a bound on the number of zero $s$ of $f(s, t)$ inside a disk of radius $r$ in complex $t$ plane. Thus we arrive at

Lemma 1. The number $n_{r}(s)$ of $f(s, t)$ within the disk $|t|<r<t_{0}-\varepsilon=R$ is bounded from above by

$$
n_{r}(s) \leqq \frac{e r^{1 / 2}}{2 \sqrt{R}} \ln \bar{s} .
$$

Proof. According to Jensen's theorem [9] $n_{r}(s)$ satisfies the inequality

$$
\begin{aligned}
& n_{r}(s) \leqq \frac{1}{\ln (1 / \delta)} \operatorname{Max} \ln \left|\frac{f(s, r / \delta)}{f(s, o)}\right|, \quad \frac{r}{R}<\delta<1, \\
& n_{r}(s)<\frac{1}{\ln (1 / \delta)} \operatorname{Max}|\ln f(s, r / \delta)|,
\end{aligned}
$$

since $f(s, o)=1$. Using (5) in (8) we obtain

$$
n_{r}(s) \leqq \frac{1}{\ln (1 / \delta) \sqrt{R}}(\ln \bar{s})(r / \delta)^{1 / 2} .
$$

Optimizing (9) with respect to $\delta$ we deduce the desired result (6). Q.E.D.

Corollary. $f(s, t)$ has no zeros inside a shrinking disk

$$
r_{0}<\frac{4 R}{e^{2}(\ln \bar{s})^{2}}
$$

Remark. $r_{0}$ shrinks as $(\ln \bar{s})^{-2}$; this result is similar to that of Eden and Kaiser [10] who studied distribution of zeros of the scattering amplitudes that violate Pomeranchuk theorem.

Definition. We define the slope of the diffraction peak, $b(s)$, and the curvature of the diffraction peak, $c(s)$, as follows.

$$
\begin{aligned}
& \left.b(s) \equiv \frac{d}{d t} f(s, t)\right|_{t=0} \\
& \left.c(s) \equiv \frac{d^{2}}{d t^{2}} \ln f(s, t)\right|_{t=0} .
\end{aligned}
$$

Lemma 2. The slope and curvature of the diffraction peak defined through Equations (11a) and (11b) respectively, are bounded from above as

$$
\begin{aligned}
& b(s) \leqq \frac{e^{2}}{4 R}(\ln \bar{s})^{2}, \\
& c(s) \leqq \frac{3 e^{2}}{16 R^{2}}(\ln \bar{s})^{4} .
\end{aligned}
$$


Proof. Let us apply Cauchy's inequality to $b(s)$. Then

$b(s) \leqq \frac{1}{|t|} \operatorname{Max}|f(s, t)|<R$

Using (5) in (14) and then optimizing with respect to $t$, we arrive at (12).

Moreover,

$\left.\frac{d^{2}}{d t^{2}} f(s, t)\right|_{t=0} \leqq \frac{2}{|t|^{2}} \underset{|t|<R}{\operatorname{Max}}|f(s, t)|$.

Optimizing with respect to $|t|$ we have

$$
\left.\frac{d^{2}}{d t^{2}} f(s, t)\right|_{t=0} \leqq \frac{e^{4}}{8 R^{2}}(\ln \bar{s})^{4}
$$

Thus

$$
\begin{aligned}
C(s) & \leqq \frac{e^{4}}{8 R^{2}}(\ln \bar{s})^{4}+b^{2}(s) \\
& \leqq \frac{3 e^{4}(\ln \bar{s})^{4}}{16 R^{2}} . \quad \text { Q.E.D. }
\end{aligned}
$$

In what follows, we shall show an intimate connection between distribution of zeros of $f(s, t)$ and the slope of the diffraction peak and its curvature. It is possible to improve (14) and (15) if $f(s, t)$ is zero free inside a disk around $t=o$. The result is stated below as

Lemma 3. If $f(s, t)$ is free from zeros inside the disk $t<r(s)$, then the slope of the diffraction peak, $b(s)$, is bounded from above as

$$
\begin{aligned}
& b(s) \leqq \frac{2 \ln \bar{s}}{(r(s) R)^{1 / 2}} \\
& c(s) \leqq \frac{8 \ln \bar{s}}{r^{3 / 2} R^{1 / 2}} .
\end{aligned}
$$

Proof. Before proceeding further one has to consider two cases; (a) $r(s)<t_{0}$ and (b) $r(s) \geqq t_{0}$. Let us proceed with the first case.

Define

$$
\left.D_{n}(s) \equiv \frac{1}{n !} \frac{d^{n}}{d t^{n}} \ln f(s, t)\right|_{t=0} .
$$

Note that

$$
D_{1}(s)=b(s) \text {. }
$$

Since $f(s, t)$ is free of zeros inside the disk $|t|<r(s), f(s, t)$ is holomorphic in this domain. Applying Cauchy's inequality to $D_{n}(s)$ we get

$$
D_{n}(s) \leqq \frac{1}{|\varrho|^{n}} \max _{|t|=\varrho}|\ln f(s, \varrho)|,|\varrho|<r(s)<t_{0} .
$$


In order to determine $\max |\ln f(s, \varrho)|$ for $|\varrho|<r(s)$ we apply Borel-Carathéodory theorem [11] to $f(s, t)$.

$$
|\ln f(s, \varrho)| \leqq \frac{2|\varrho|}{r-|\varrho|} \operatorname{Max}_{|t|=r(s)}|\ln f(s, r)|
$$

since $f(s, o)=1$. Using (5) in (21) we get

$$
|\ln f(s, \varrho)| \leqq \frac{2|\varrho| r(s)}{r-|\varrho|} \frac{\ln \bar{s}}{\sqrt{R}} .
$$

Thus upper bound (20) on $D_{n}(s)$ is

$$
D_{n}(s) \leqq \frac{2 \sqrt{r(s)}}{|\varrho|^{n-1}(r-|\varrho|)} \frac{\ln \bar{s}}{\sqrt{R}} .
$$

Optimizing with respect to $\varrho$ we get

$$
\begin{aligned}
& b(s) \leqq \frac{2 \ln \bar{s}}{(r(s) R)^{1 / 2}}, \\
& c(s) \leqq \frac{8 \ln (\bar{s})}{r(s)^{3 / 2} R^{1 / 2}} .
\end{aligned}
$$

(b) If $r(s) \geqq t_{0}$ then we can apply Borel-Caratheodory inequality to the bigger circle $|t|=R$ and obtain bounds better than (24) and (25). Q.E.D.

Remarks. (a) If $r(s)$ is independent of $s$ then $b(s)$ and $c(s)$ are bounded by $\ln \bar{s}$.

(b) However, if $r(s)>(\ln \bar{s})^{-2+\delta}, \delta>0$ then (24) and (25) will be improvements over (12) and (13).

Corollary. If $r(s)$ is independent of $s$ the Froissart bound can be improved as follows

$$
\sigma_{\mathrm{tot}}(s) \leqq \frac{\ln \bar{s}}{(R r)^{1 / 2}} .
$$

Proof. We follow the method due to Eden and Kaiser [10] to prove the above result. It follows from (21) that

$$
|\ln f(s, \varrho)| \leqq \frac{2|\varrho|}{r-|\varrho|} \max _{|\varrho|<r}|\ln f(s, \varrho)| .
$$

For $\varrho<0$, therefore,

$$
\frac{d \sigma^{A}}{d t}(s, t)>\frac{d \sigma^{A}}{d t}(s, o) \exp 2 t(r R)^{-1 / 2} \ln \bar{s}
$$

for $r>|t| / 2$ which follows from standard theorem [12]. Therefore

$$
\sigma_{\mathrm{tot}}>\sigma_{\mathrm{el}}>\int \frac{d \sigma^{A}}{d t} d t>\int_{-r / 2}^{0} \frac{d \sigma^{A}}{d t}(s, o) \exp \left(2 t(r R)^{-1 / 2} \ln \bar{s}\right) d t .
$$


Hence

$$
\sigma_{\text {tot }}>\frac{\sigma_{\text {tot }}^{2}(R r)^{1 / 2}}{\ln \bar{s}}\left(1-\exp \left(-\ln \bar{s} / R^{1 / 2}\right)\right)
$$

since $\frac{d \sigma^{A}}{d t}(s, t) \geqq \sigma_{\text {tot }}^{2}$. Therefore

$$
\sigma_{\text {tot }} \leqq \ln \bar{s} /(r R)^{1 / 2}
$$

for large enough s. Q.E.D.

\section{Distribution of Zeros of $f(s, t)$ and Scaling of Diffraction Peak}

In this section we investigate how the energy dependence of $b(s)$ affects the distribution of zeros of $f(s, t)$. Let us assume that $f(s, t)$ has no zeros in $|t|<r, r$ being independent of $s$, and $b(s)$ behaves as

$$
b(s)=C(\ln \bar{s})^{\alpha} \quad 0<\alpha \leqq 2
$$

$C$ being a constant. Then it follows from (16) that

$$
r<\frac{4(\ln \bar{s})^{2(1-\alpha)}}{C^{2} R}
$$

For $\alpha>1$ this is in contradiction with the $s$-dependence of $r$. Thus when $1<\alpha \leqq 2$ $f(s, t)$ must have at least a pair of zeros in the disk

$$
r<\frac{4}{C^{2} R}(\ln \bar{s})^{2(1-\alpha)} .
$$

Note that the radius of the disk tends to zero for $s \rightarrow \infty$. Further, one can follow the argument due to Kinoshita and Martin [13] that all zeros of $f(s, t)$ cannot lie in the region $\operatorname{Re} t>0$. In that case unitarity bound will be violated.

Therefore, it is very interesting to note that energy dependence of $b(s)$ and unitarity provide useful information regarding location of zeros of $f(s, t)$.

The following theorem provides an interesting relation between $b(s)$ and $c(s)$ if the zeros of $f(s, t)$ are located in a specific manner.

Theorem. If $b(s) \simeq(\ln \bar{s})^{\alpha}$ such that $5 / 4<\alpha \leqq 2$ and all zeros of $f(s, t)$ lie in a domain $\left|\operatorname{Im} t_{i}\right|<\varepsilon_{1}\left|t_{i}\right|^{2}, \varepsilon_{1}$ being an arbitrarily small positive number, then

$$
\left.\frac{c(s)}{b^{2}(s)} \simeq 0(\ln s)^{2 \alpha-4}\right) \text {. }
$$

Proof. We shall prove the theorem in several steps.

i) Let us consider a disk of radius $r$ in the $t$-plane. The function

$$
\tilde{f}(s, t)=\prod_{i=1}^{n_{r}} \frac{t_{i} t_{i}^{*}}{\left(t_{i}-t\right)\left(t_{i}^{*}-t\right)} f(s, t)
$$

is free from zeros inside the disk $|t|<r$; where $t_{i}$ are the zeros of $f(s, t)$ in that domain and $n_{r}$ denotes the number of zeros in that disk. Note that if $t_{i}$ is a zero of $f(s, t)$ so is 
$t_{i}^{*}$. Let us define

$$
\tilde{C}_{n}(s)=\left.\frac{1}{n !} \frac{d^{n}}{d t^{n}} \ln f(s, t)\right|_{t=0}
$$

Then

$$
\tilde{C}_{n}(s)=D_{n}(s)+\frac{1}{n} \sum_{i=1}^{n_{r}} \frac{t_{i}^{n}+t_{i}^{* n}}{\left|t_{i}\right|^{2 n}} .
$$

ii) Since $f(s, t)$ is zero free inside the disk $|t|<r$ we can deduce following bounds on $C_{n}(s)$

$$
\left|\tilde{C}_{n}(s)\right| \leqq \frac{1}{|\varrho|^{n-1}(r-|\varrho|)} \underset{|\varrho|=r}{\operatorname{Max}}|\ln f(s, \varrho)|
$$

which is analog of (23). We have to deduce an upper bound on $\ln f(s, t)$.

iii) Let $n_{r}^{\prime}$ denote the number of zeros of $f(s, t)$ within the disk $r / 2<|t|<r$. Our purpose is to find an upper bound on [14]

$$
\begin{aligned}
& \prod_{i} \frac{t_{i} t_{i}^{*}}{\left(t_{i}-t\right)\left(t_{i}^{*}-t\right)} f(s, t) \quad \text { for } \quad|t|<r . \\
& \operatorname{Max}_{|t|=r+\varepsilon}\left|\prod_{i}^{n_{r}^{\prime}} \frac{t_{i} t_{i}^{*}}{\left(t_{i}-t\right)\left(t_{i}^{*}-t\right)}\right|<\left(\frac{r}{\varepsilon}\right)^{n_{r}^{\prime}},
\end{aligned}
$$

where the product is over the zeros in the region $r / 2<|t|<r$. For the zeros in the region $0<|t| \leqq r / 2$ we have

$$
\begin{aligned}
& \underset{|t|=r+\varepsilon}{\operatorname{Max}}\left|\prod_{i}^{\prime \prime} \frac{t_{i} t_{i}^{*}}{\left(t_{i}-t\right)\left(t_{i}^{*}-t\right)} f(s, t)\right| \\
& \leqq\left(\frac{r / 2}{r / 2+\varepsilon}\right)^{n_{r}-n_{r}^{\prime}} \exp \left[\frac{(\ln \bar{s}) \sqrt{r+\varepsilon}}{\sqrt{R}}\right] \\
& \quad \leqq \exp \frac{\ln \bar{s} \sqrt{r+\varepsilon}}{\sqrt{R}} .
\end{aligned}
$$

Thus

$$
\underset{|t|=r+\varepsilon}{\operatorname{Max}}|\tilde{f}(s, t)| \leqq\left(\frac{r}{\varepsilon}\right)^{n_{r}^{\prime}} \exp \left[(\ln \bar{s})\left(\frac{r+\varepsilon}{R}\right)^{1 / 2}\right] .
$$

Optimizing (40) with respect to $\varepsilon$ we get

$$
\begin{aligned}
\operatorname{Max}|\ln \tilde{f}(s, t)| \leqq & {\left[n_{r}^{\prime} \ln \left\{\left(\frac{r}{R}\right)^{1 / 2} \ln \bar{s}\right\}\right.} \\
& -n_{r}^{\prime} \ln \left(n_{r}^{\prime}+2\right) \\
& \left.+r R^{-1 / 2} \ln \bar{s}\right] .
\end{aligned}
$$


If we apply the same technique to (37) as applied to (23) we arrive at

$$
\begin{aligned}
\tilde{C}_{n}(s) \leqq & \frac{2}{r^{n}\left(\frac{n-1}{n}\right)^{n-1}}\left[n_{r}^{\prime} \ln \left(R^{-1 / 2} r^{1 / 2} \ln \bar{s}\right)\right. \\
& \left.+r^{1 / 2} R^{-1 / 2} \ln \bar{s}\right] . \quad n \geqq 2 .
\end{aligned}
$$

iv) From (42) we note

$$
\begin{aligned}
& \tilde{C}_{1}(s) \leqq 2(r R)^{-1 / 2} \ln \bar{s}+n_{r}^{\prime} \ln \left(R^{-1 / 2} \ln \bar{s}\right) \\
& C_{2}(s) \leqq r^{-3 / 2} R^{-1 / 2} \ln \bar{s}+n_{r}^{\prime}(s) \ln \left(R^{-1 / 2} \ln \bar{s}\right) .
\end{aligned}
$$

Rewriting Equation (36) for $n=2$ gives

$$
\tilde{C}_{2}(s)=D_{2}(s)+\sum_{i=1}^{n_{r}} \frac{t_{i}^{2}+t_{i}^{* 2}}{2\left|t_{i}\right|^{4}} .
$$

Under the assumption of the theorem the second term of the right hand side of (44) satisfies

$$
0<\sum \frac{t_{i}^{2}+t_{i}^{* 2}}{\left|t_{i}\right|^{4}} \simeq 0(\ln \bar{s})^{4(\alpha-1)},
$$

where as the left hand side is bounded from above by (43). Thus, in order to satisfy (44) $D_{2}(s)<0$ and should be $0(\ln \bar{s})^{4(\alpha-1)}$. Since $b(s) \simeq(\ln \bar{s})^{\alpha}$ is follows that

$$
\frac{c(s)}{b(s)^{2}} \simeq 0(\ln \bar{s})^{(2 \alpha-4)} .
$$

This completes the proof of the theorem. Q.E.D.

Remark. Note that $5 / 4<\alpha \leqq 2$. Therefore i) $c(s) / b^{2}(s) 0(\ln \bar{s})^{-3 / 2}$ when $\alpha \simeq 5 / 4$ and ii) $c(s) / b^{2}(s) \simeq 0(1)$ for $\alpha=2$. $c(s) / b^{2}(s) \rightarrow 0$ in the first case and it is bounded by a constant in the second case. It has been pointed out by Cornille and Martin [5] that a sufficient condition for scaling of diffraction peak is $c(s) / b^{2}(s)<$ const with the scaling variable $\tau=t b(s)$. Here by scaling we mean that

$$
f(s, \tau / b(s)) \underset{s \rightarrow \infty}{\longrightarrow} f(\tau)
$$

for at least one sequence $\left\{s_{n}\right\} \rightarrow \infty$ and $\tau=t b(s)$. We mention here that the hypothesis of our theorem provides a sufficient condition for scaling of diffraction peak.

To summarize we have deduced several bounds on the slope of the diffraction peak, $b(s)$, and the curvature of the diffraction peak, $c(s)$, and have investigated the distribution of zeros of $f(s, t)$. Finally, we have shown that energy dependence of $b(s)$ and the location of zeros of $f(s, t)$ in complex $t$ plane impose constrains on ratio between curvature and slope of the diffraction peak for absorptive scattering cross sections. 


\section{References}

1. Epstein,H., Glaser,V., Martin, A.: Commun. math. Phys. 13, 257 (1969)

2. For review articles on theorems on high energy collisions Eden, R.J.: Rev. Mod. Phys. 43, 15 (1971)

Roy, S. M.: Phys. Rep. C 5, 125 (1972)

3. Froissart, M.: Phys. Rev. 123, 1053 (1961)

Lukaszuk,L., Martin, A. : Nuovo Cimento 52 A, 122 (1967)

4. Auberson, G., Kinoshita, T., Martin, A. : Phys. Rev. D 3, 3185 (1971)

5. Cornille,H., Martin, A. : Nucl. Phys. B 115, 163 (1976)

6. Cornille,H.: Phys. Rev. D 14, 1693 (1976) Auberson, G., Roy,S. M.: Nucl. Phys. B 117, 322 (1977)

For an excellent review see

Martin, A.: Scaling and spin effects in diffraction peak. CERN Preprint, TH-2229

7. Mahoux, G.: Phys. Letters 65 B, 139 (1976)

8. Roy,S.M.: TIFR Preprint TIFR/TH/77-24 (1977)

9. Titchmarsh,E.C.: The theory of function, p. 171. London: Oxford University Press 1939

10. Eden, R.J., Kaiser, G.D. : Nucl. Phys. B 28, 253 (1971)

For distribution of zeros of the amplitudes see

Bessis,J.D.: Nuovo Cimento 45 A, 974 (1966)

11. Reference [9], p. 174

12. Boas, R.P.: Entire functions. New York: Academic Press 1945, Theorem 1.2.3, p. 3

13. Kinoshita, T., Martin, A. : (unpublished), quoted in footnotes of [4]

14. Here we closely follow

Kinoshita,T., Maharana,J.: J. Math. Phys. 16, 2294 (1975)

Communicated by R. Haag

Received September 3, 1977 
